\title{
Fauna parasitaria en caninos (Canis lupus familiaris) de un sector rural de la región central de Chile
}

\author{
Parasitic fauna in dogs (Canis lupus familiaris) of a rural sector in the \\ central region of Chile
}

\author{
Alvaro Opazo ${ }^{1,4}$, Carlos Barrientos ${ }^{2}$, Ana María Sanhueza ${ }^{2}$, \\ Nicole Urrutia ${ }^{2}$, Italo Fernández ${ }^{3}$
}

\section{Resumen}

El objetivo de este trabajo fue determinar la presencia de ecto y endoparásitos en una población canina ubicada en un sector rural montañoso de la región de Valparaíso, Chile. Treinta caninos fueron sometidos a inspección clínica para recolectar ectoparásitos y heces del recto. Las muestras de heces fueron analizadas mediante la técnica coproparasitaria de Burrows y el Test SNAP ${ }^{\circledR}$ Giardia (IDEXX). E1 73\% de los caninos presentaron endoparasitismo a la técnica coproparasitaria. Se identificaron los helmintos Toxocara canis (40\%), Strongyloides stercoralis (17\%), Dipylidium caninum (17\%), Uncinaria stenocephala (13\%), Ancylostoma caninum (7\%) Trichuris vulpis (3\%), y los protozoos Isospora sp (13\%), Sarcocystis sp (3\%), Entamoeba coli (3\%) y Blastocystis $\mathrm{sp}(3 \%)$. La prevalencia de Giardia sp fue de $17 \%$, pero mediante el SNAP $®$ Giardia fue de $27 \%(\mathrm{p}=0.0004)$. Se encontraron garrapatas en 25 caninos $(83 \%)$, identificándose las especies Rhipicephalus sanguineus y Amblyomma tigrinum. Así mismo, 10 caninos (33\%) presentaron pulgas, identificándose las especies Ctenocephalides felis felis, Ctenocephalides canis y Pulex irritans.

Palabras clave: parásitos; perros; ambiente rural; zoonosis; Giardia

\section{Abstract}

The aim of this study was to determine the presence of ecto- and endoparasites in a canine population located in a mountainous rural sector of the Valparaíso region of Chile. Thirty canines were subjected to clinical inspection to collect ectoparasites and

\footnotetext{
${ }^{1}$ Universidad Andres Bello, Escuela Medicina Veterinaria, Facultad Ciencias de la Vida, Concepción, Chile

${ }^{2}$ Facultad de Recursos Naturales y Medicina Veterinaria, Universidad Santo Tomás, Concepción, Chile

${ }^{3}$ Facultad de Ciencias Biológicas, Universidad de Concepción, Concepción, Chile

${ }^{4}$ E-mail: alvaro.opazo@unab.cl
}

Recibido: 11 de mayo de 2018

Aceptado para publicación: 31 de octubre de 2018 
faeces of the rectum. Faecal samples were analysed using the Burrow's coproparasitic technique and the SNAP ${ }^{\circledR}$ Giardia Test (IDEXX). Results showed that $73 \%$ of canines had endoparasites by the coproparasitic technique. The helminths Toxocara canis (40\%), Strongyloides stercoralis (17\%), Dipylidium caninum (17\%), Uncinaria stenocephala (13\%), Ancylostoma caninum (7\%) Trichuris vulpis (3\%), and the protozoa Isospora sp $(13 \%)$, Sarcocystis sp (3\%), Entamoeba coli (3\%) and Blastocystis sp (3\%). The prevalence of Giardia sp was $17 \%$, but it was $27 \%$ by the SNAP ${ }^{\circledR}$ Giardia ( $\left.\mathrm{p}=0.0004\right)$. Ticks were found in 25 canines (83\%), identifying the species Rhipicephalus sanguineus and Amblyomma tigrinum. Likewise, 10 dogs (33\%) presented fleas, identifying the species Ctenocephalides felis felis, Ctenocephalides canis and Pulex irritans.

Key words: parasites; dog; rural environment; zoonosis; Giardia

\section{INTRODUCCIÓN}

El perro (Canis lupus familiaris) es un reconocido hospedero de parásitos internos y externos que están relacionados a procesos infecciosos de relevancia clínica. Por ejemplo, parásitos intestinales, tanto protozoarios como helmintos, son uno de los principales causantes de patologías gastrointestinales, especialmente en animales jóvenes y neonatos (Irwin 2002; López et al., 2006). De igual manera, ectoparásitos, tales como garrapatas, piojos y pulgas, están relacionados con procesos clínicos tanto en mascotas como en seres humanos, favoreciendo la ocurrencia de infecciones de diversa etiología (Irwin, 2002). Así mismo, algunos de estos parásitos son responsables de importantes infecciones de carácter zoonótico, incluyendo parasitosis bien documentadas como hidatidosis, síndrome de larva migrans cutánea y visceral, y sarna sarcóptica, así como otras infecciones reemergentes como rickettsiosis, criptosporidiasis y giardiasis (Acha y Szyfres, 2003).

La ocurrencia de estas parasitosis en el perro muestra diferencias importantes, tanto en prevalencia como intensidad de infección, dependiendo del lugar en donde estos estudios han sido efectuados. Esto se debería a que el parasitismo está influenciado por factores tales como presencia de dueño, tipo de hábitat, clima y cohabitación con otros hospederos que, de una u otra manera, reflejan las condiciones particulares en los cuales se desenvuelven estos animales (Barriga, 2002).

En los últimos veinte años, diversas investigaciones efectuadas en Chile han dado cuenta de la presencia de parasitismo en el perro, aunque la mayoría analiza ejemplares de hábitats urbanos y relacionados con contaminación fecal en espacios públicos (Gorman et al., 2006; López et al., 2006; Armstrong et al., 2011; Luzio et al., 2017), pero las referencias bibliográficas de esta problemática en ecosistemas rurales son escasas (Acosta-Jamett et al., 2010; Abarca et al., 2016). Debido a esto, y teniendo en consideración que esta información es esencial para la elaboración y ejecución de medidas de control en salud pública y animal, se procedió a efectuar este estudio, cuyo objetivo fue determinar la presencia de parásitos externos e internos de perros que habitan un sector rural de la Región de Valparaíso, zona central de Chile.

\section{Materiales y Métodos}

\section{Zona Geográfica}

Se realizó un estudio descriptivo y transversal dentro de un área geográfica de alrededor de $9.46 \mathrm{~km}^{2}$, en un sector rural ubica- 
do a $90 \mathrm{~km}$ al noroeste de Santiago, en la región de Valparaíso, zona central de Chile. Este es un sector montañoso de la Cordillera de la Costa con una altitud entre 787 a 2200 msnm, con caminos de tierra, angostos, que atraviesan diversos cerros y colinas, con clima mediterráneo con inviernos suaves y veranos cálidos y secos. En este sector habitan familias de nivel socioeconómico bajo, dedicados a la producción agropecuaria de subsistencia, teniendo principalmente bovinos, equinos y cabras, donde sus perros cumplen funciones de guardianía y compañía. Además, la fauna nativa del lugar la conforman diversas aves, zorros y conejos. El sector donde se realizó el muestreo se ubica próximo a dos grandes núcleos urbanos, Santiago y Viña del Mar, existiendo parcelas con casas utilizadas para el descanso en los fines de semana y vacaciones por familias y mascotas (perros y gatos) que provienen de esas ciudades.

\section{Tamaño Muestral y Muestras}

Se realizó un muestreo aleatorio a 30 caninos que habitan en ese sector rural, mediante recorrido en vehiculo $4 \times 4$, reclutando canes, indistintamente del sexo, los cuales cumplieron los siguientes criterios de inclusión: residencia por más de un año en este sector y firma del consentimiento informado por un responsable del canino. A cada perro seleccionado y en la misma vivienda se le realizó un examen clínico general y se le extrajeron ectoparásitos si eventualmente los presentaban. Durante el examen físico del canino, se inspeccionaron las zonas anatómicas donde suelen adherirse las garrapatas (cabeza, orejas, zona interdigital, etc.), extrayéndolas con unas pinzas gancho para quitar garrapatas (Grupanor Cercampo®). En el caso de las pulgas, la búsqueda se iniciaba en la zona dorso-lumbar para observar signos de dermatitis alérgica por picadura de pulgas (DAPP) o heces de pulgas (utilizando linternas y lupas manuales), y luego en posición decúbito dorsal revisando la zona abdominal. Las pulgas fueron recogidas de manera manual, siempre con la utilización de guantes de látex. Los ectoparásitos fueron almacenados en tubos Eppendorf con alcohol al 70\% (Ybañez et al., 2012).

Se colectaron muestras de heces mediante tacto rectal y fueron guardadas en frascos con solución PAF (fenol, paraformaldehido y alcohol) y en frascos sin solución conservante para realizar el Test Snap ${ }^{\circledR}$ Giardia de IDEXX. Todas las muestras fueron individualizadas con el número del paciente $\mathrm{y}$ almacenadas a temperatura de refrigeración $\left(4^{\circ} \mathrm{C}\right)$ en una caja térmica y al final del día conservadas en refrigerador $\left(4^{\circ} \mathrm{C}\right)$. Además, se recabó información sobre sexo, edad y raza, así como antecedentes epidemiológicos generales consultados al dueño del animal como, por ejemplo, tipo de alimentación, acceso libre o restringido al exterior de la propiedad, existencia previa o no de desparasitación y/o vacunación previa, entre otros.

\section{Procesamiento de las Muestras}

Los ectoparásitos recolectados fueron identificados por observación directa mediante lupa estereocópica (Kyoto Optical, XTJ$4400 ; 4 \mathrm{x}) \mathrm{y}$ revisión de literatura especializada de referencia (Alcaíno et al., 2002; Linardi y Santos, 2012; Nava et al., 2017).

El procesamiento de las muestras de heces se realizó en el Laboratorio de Parasitología de la Universidad Santo Tomás, Concepción, Chile, mediante la técnica coproparasitaria de Burrows (Burrows, 1967). La identificación taxonómica se efectuó mediante el uso de microscopía óptica (Olympus, CX21; 100x y 400x) y literatura especializada de referencia (Borchet, 1974; Soulsby, 1987; Blanco y Galiano, 1989). Las muestras de heces frescas fueron analizadas mediante el test de ELISA SNAP® Giardia de IDEXX según protocolo del fabricante. Se realizó la prueba de concordancia con el índice de Kappa y el test exacto de Fischer entre los resultados de Giardia identificados por la técnica coproparasitaria y el SNAP ${ }^{\circledR}$ Giardia. 
Cuadro 1. Características biométricas y descripción epidemiológica de la población canina rural de la región de Valparaíso en Chile ( $\mathrm{n}=30$ perros)

\begin{tabular}{llcc}
\hline Sexo & $93 \%$ machos & $7 \%$ hembras & \\
Esterilizado & $3.3 \%$ machos & 0 hembras & \\
Edad (media $\pm \mathrm{DE})$ & $56.0 \pm 32.7$ meses. De adulto jóvenes a geriátricos & \\
Tipo racial & $80 \%$ mestizos & $20 \%$ de raza & \\
Condición corporal & $33 \% 2$ de 5 & $60 \% 3$ de 5 & $7 \% 4$ de 5 \\
Alimentación & $20 \%$ comercial & $16.6 \%$ casera & $63.3 \%$ ambas \\
Acceso exterior de la & $100 \%$ con acceso al exterior, deambulando libremente por el sector \\
vivienda & rural & & \\
Vacunación & $3.3 \%$ con vacunación (séxtuple) \\
Desparasitación & $73.3 \%$ desparasitación externa realizada por la municipalidad una \\
& vez al año & \\
\hline
\end{tabular}

Los resultados fueron expresados en frecuencia relativa (prevalencia) y frecuencia absoluta, de acuerdo con las definiciones consideradas por Bush et al. (1997).

\section{Resultados}

El análisis de los datos epidemiológicos obtenidos del responsable del canino permitió observar que el manejo zoosanitario de los caninos era deficiente (Cuadro 1).

\section{Ectoparásitos}

Se recolectaron 93 garrapatas y 23 pulgas. En el 83\% (25/30) de los canes se encontró garrapatas y en el $33 \%(10 / 30)$ se encontró pulgas. El 88.1\% (82/93) de las garrapatas era Rhipicephalus sanguineus sensu lato y el 11.5\% (11/93) correspondió a Amblyomma tigrinum. El 56\% (46/82) de las garrapatas $R$. sanguineus eran hembras; así mismo, el 81\% (67/93) eran adultos y el $19 \%$ restante eran ninfas. En el caso de $A$. tigrinum, el 36\% (4/11) fueron hembras y el $64 \%$ restante era machos, habiéndose encontrado solo ejemplares adultos.
En el caso de las pulgas, 43\% (10/23) fue de la especie Ctenocephalides felis felis, $39 \%(9 / 23)$ Ctenocephalides canis y 17\% (4/23) Pulex irritans. La identificación se hizo en vista lateral de la cabeza y cuerpo del ectoparásito.

\section{Endoparásitos}

El 73\% (22/30) de los caninos fueron positivos a parásitos intestinales. Se identificaron los helmintos Toxocara canis, Strongyloides stercoralis, Dipylidium caninum, Uncinaria stenocephala, Ancylostoma caninum, Trichuris vulpis $\mathrm{y}$ los protozoos Isospora sp, Sarcocystis sp, Entamoeba coli, Blastocystis sp y Giardia sp (Cuadro 2). La prevalencia de Giardia obtenida mediante ELISA (27\%) resultó mayor que la observado a través de la técnica de Burrows (17\%), lo que evidencia que la técnica de ELISA fue más sensible. Al considerar como prueba estándar al método SNAP® Giardia, se obtuvo una concordancia del 0.7097 (índice de Kappa) que, según la escala de valoración, el grado de concordancia se clasificó como «bueno» (entre 0.6 y 0.8) (Viera y Garrett, 2005). La diferencia entre las dos pruebas fue en tres perros que 
Cuadro 2. Especies parasitarias encontradas mediante la técnica de Burrows en la población canina rural de la región de Valparaíso, Chile ( $\mathrm{n}=30$ perros)

\begin{tabular}{lc}
\hline & $\begin{array}{c}\text { Prevalencia } \\
(\%)\end{array}$ \\
\hline Helmintos & 40 \\
Toxocara canis & 17 \\
Strongyloides stercoralis & 17 \\
Diphilydium caninum & 13 \\
Uncinaria stenocephala & 7 \\
Ancilostoma caninum & 3 \\
Trichuris vulpis & \\
Protozoos & 17 \\
Giardia sp & 13 \\
Isospora sp & 3 \\
Sarcocystis $\mathrm{sp}$ & 3 \\
Entamoeba coli & 3 \\
Blastocystis sp & \\
\hline
\end{tabular}

resultaron positivos al SNAP ${ }^{\circledR}$ Giardia pero que fueron negativos a la técnica de Burrows.

\section{Coinfestación y Coinfección}

El 44\% (11/25) de los caninos parasitados presentaron garrapatas y pulgas, el $56 \%$ $(14 / 25)$ solo tuvo garrapatas, y ningún canino tuvo solamente pulgas. De los 25 caninos con garrapatas, solo el $24 \%(6 / 25)$ estaba parasitado por $R$. sanguineus sensu lato y A. tigrinum. Respecto a las pulgas, de los 10 caninos infestados, el 20\% (2/10) presentó coinfestación con C. felis y C. canis, $20 \%$ con $C$. canis y $P$. irritans y $10 \%$ (1/10) estuvo infestado con las tres especies de pulgas.

Con relación a los endoparásitos, el 27\% (6/22) de los canes parasitados presentaron coinfección de helmintos y protozoos, siendo la mayor asociación entre Giardia sp, $T$. canis y $S$. stercolaris $(14 \%, 3 / 22)$. Dentro de los helmintos, el 53\% (9/17) presentó biparasitismo, encontrándose más frecuente la asociación entre $T$. canis y $S$. stercoralis en cuatro caninos. Por último, de los 11 caninos positivos a protozoos, $91 \%(10 / 11)$ presentó monoparasitismo y el 10\% (1/10) presentó biparasitismo, principalmente con la asociación Isospora sp y Blastocystis sp.

\section{Discusión}

El ambiente rural se presenta como un escenario propicio para la presencia, desarrollo y diseminación de distintos parásitos, muchos de ellos con potencial zoonótico y con consecuencias graves para la salud humana. Los resultados de esta investigación ratifican la presencia de parásitos, algunos de reconocida importancia en salud pública como $T$. canis y Giardia sp, así como ectoparásitos que pueden ser transmisores de diversos patógenos (Irwin, 2002; López et al., 2006; Rishniw et al., 2006; Jiménez-Coello et al., 2010; Abarca et al., 2013; Rocha et al., 2013; Cicuttin et al., 2014).

Independiente del objetivo para el cual se tenga un canino (guardia, compañía o terapia asistida), la Ley Chilena N. ${ }^{\circ} 21.020$ de tenencia responsable de mascotas y animales de compañía (2017) impone una serie de obligaciones al propietario, entre las cuales está proveerle de vacunaciones y desparasitaciones, de una alimentación adecuada y de mantenerlo confinado en la vivienda o sacarlo al exterior con un arnés evitando que deambulen libremente. En este estudio se evidenció que todos los perros deambulan libremente, solo el 3.3\% está vacunado y el $27 \%$ no ha sido desparasitado. Además, la desparasitación externa solo ocurre una vez al año en operativos sanitarios realizados por la municipalidad de la zona.

Los parásitos identificados en esta investigación ya han sido reportados en perros de Chile (Tagle 1966; Alcaino y Gorman, 1999). Las especies de garrapatas y de pul- 
gas han sido reportadas en caninos de zonas rurales y urbanas en las regiones de Arica, Coquimbo, Metropolitana y de La Araucanía (Abarca et al., 2016) y en la región Metropolitana, Bío Bío y Los Lagos (Alcaíno et al., 2002).

La mayor prevalencia de $R$. sanguineus $(88 \%)$ coincide con lo registrado en la literatura respectiva de Chile (González-Acuña y Guglielmone, 2005; Abarca et al., 2016) y en el mundo (Dantas-Torres, 2010). Por el contrario, A. tigrinum presentó una menor prevalencia y solo ejemplares adultos, lo que concuerda con lo reportado en estudios similares, donde se indica que los estadios juveniles se alimentan en roedores y aves (Guglielmone et al., 2000; González-Acuña et al., 2004; Nava et al., 2006) y no en caninos. En el trabajo de revisión de Dantas-Torres (2010), $R$. sanguineus se le vinculado con Babesia vogeli, Ehrlichia canis, Hepatozoon canis, Rickettsia coronii, Rickettsia massiliae y $R$. rickettsii. Además, Cicuttin et al. (2014) incluye en ese listado a Rickettsia massiliae y Anaplasma platys. En Chile, no se ha evidenciado la presencia $R$. massiliae, B. vogeli, $H$. canis, $R$. coronii y $R$. rickettsii, pero sí de Rickettsia felis identificada en $R$. sanguineus en el norte y centro de Chile (Abarca et al., 2013a) y de Rickettsia andeanae aislada por primera vez en garrapatas $A$. tigrinum en el norte y centro sur en Chile, aunque sin conocer aún su capacidad patológica en animales o humanos (Abarca et al., 2013b).

En el caso de las pulgas y debido a sus cualidades sinantrópicas, distribución cosmopolita y variedad de especies, tienen gran importancia como vectores de enfermedades zoonóticas. Se han identificado bacterias del género Bartonella, Rickettsia (R. felis, $R$. prowazekii, $R$. typhi), Yersinia pestis, así como el céstodo Dipylidium caninum, entre otros agentes infecciosos en $C$. felis felis, $C$. canis y P. irritans (Bitam et al., 2010). En Chile, no hay estudios publicados respecto de la capacidad vectora de las pulgas, siendo abocados a su identificación morfológica, $\mathrm{y}$ que concuerdan con los hallazgos del presente estudio (Alcaíno y Gorman, 1999; Alcaíno et al., 2002).

Se debe mencionar que para aumentar la sensibilidad de los exámenes coproparasitarios es deseable tomar tres muestras por animal, debido a la liberación intermitente de formas parasitarias diagnósticas (Otranto, 2015), lo que en este estudio no fue posible de efectuar. Interesante fue el hallazgo de larvas rabditoides de $S$. stercoralis en esta población, debido a que este helminto se relaciona principalmente con caninos inmunosuprimidos (Cervone et al., 2016) y personas de la misma condición aunado a malas condiciones higiénicas (Stepek et al., 2006), situación observada en algunas viviendas del presente estudio.

Destaca el hecho de que de los ocho ejemplares positivos al Test SNAP ${ }^{\circledR}$ Giardia de IDEXX, cuatro no presentaban síntomas atribuibles a Giardia, corroborando la ciclicidad de los signos clínicos de este protozoo (Irwin, 2002). Además, SNAP ${ }^{\circledR}$ Giardia fue significativamente superior $(\mathrm{p}=0.0004$; test exacto Fisher) para el diagnóstico de Giardia sp en comparación con la técnica de Burrows, corroborando lo descrito en otros trabajos sobre la mayor sensibilidad diagnóstica de los test inmuno-serológicos y por PCR (Bouzid et al., 2015). En este caso, el ELISA SNAP ${ }^{\circledR}$ Giardia de IDEXX es una prueba ampliamente utilizada para identificar el antigeno libre de Giardia en heces, presentando una sensibilidad aproximada de $92 \%$ y una especificidad del 99\%, según el fabricante (www.idexx.es/smallanimal/inhouse/ snap/giardia.html).

\section{Conclusiones}

- Los ectoparásitos de mayor prevalencia en la zona rural de Valparaíso, Chile, fueron la garrapata Rhipicepalus sanguineus sensu lato y la pulga Cteno- 
cephalides felis felis, en tanto que entre los endoparásitos, el helminto Toxocara canis y el protozoo Giardia sp fueron los más prevalentes.

- Existe una deficiente tenencia responsable en los caninos de la zona rural de Valparaíso por parte de sus propietarios, lo cual lleva al desarrollo de los parásitos encontrados, y el consiguiente riesgo para la salud pública.

\section{Literatura CitTada}

1. Abarca K, Gárate D, López J, Acosta-Jamett G. 2016. Flea and ticks species from dogs in urban and rural areas in four districts in Chile. Arch Med Vet 48: 247-253.

2. Abarca K, López J, Acosta-Jamett G, Martínez-Valdebenito C. $2013 a$. Rickettsia felis in Rhipicephalus sanguineus from two distant Chilean cities. Vector-Borne Zoonot 13: 607-609. doi: 10.1089/vbz.2012.1201

3. Abarca K, López J, Acosta-Jamett G, Martinez-Valdebenito C. 2013b. Identificación de Rickettsia andeanae en dos regiones de Chile. Rev Chil Infectol 30: 388-394. doi: 10.4067/S0716-10182013000400006

4. Acha P, Szyfres B. 2003. Zoonosis y enfermedades transmisibles comunes al hombre y a los animales. Vol III. Parasitosis. Washington DC: OPS. 413 p.

5. Acosta-Jamett G, Cleaveland S, Bronsvoort BMD, Cunningham A, Bradshaw H, Craig PS. 2010. Echinococcus granulosus infection in domestic dogs in urban and rural areas of the Coquimbo region, north-central Chile. Vet Parasitol 169: 117-122. doi: 10.1016/j.vetpar.2009.12.005

6. Alcaino H, Gorman T. 1999. Parásitos de los animales domésticos en Chile. Parasitol Día 23: 33-41. 10.4067/S071607201999000100006

7. Alcaíno HA, Gorman TR, Alcaíno R. 2002. Flea species from dogs in three cities of Chile. Vet Parasitol 105: 261-265. doi: 10.1016/S0304-4017(01)00626-4
8. Armstrong WA, Oberg C, Orellana JJ. 2011. Presencia de huevos de parásitos con potencial zoonótico en parques y plazas públicas de la ciudad de Temuco, Región de La Araucanía, Chile. Arch Med Vet 43: 127-134. doi: 10.4067/ S0301-732X2011000200005

9. Barriga OO. 2002. Las enfermedades parasitarias de los animales domésticos en la América Latina. Santiago: Ed Germinal. $247 \mathrm{p}$.

10. Bitam I, Dittmar K, Parola P, Whiting MF, Raoult D. 2010. Fleas and fleaborne diseases. Int J Infect Dis 14: 667676. doi: 10.1016/j.ijid.2009.11.011

11. Blanco J, Galiano J. 1989. Atlas de coprología: digestión y parásitos. Madrid, España: Ed Garsi. 200 p.

12. Borchet A. 1974. Parasitología veterinaria. Zaragoza, España: Acribia. 745 p.

13. Bouzid M, Halai K, Jeffreys D, Hunter PR. 2015. The prevalence of Giardia infection in dogs and cats, a systematic review and meta-analysis of prevalence studies from stool samples. Vet Parasitol 207: 181-202. doi: 10.1016/ j.vetpar.2014.12.011

14. Burrows RB. 1967. A new fixative and technics for the diagnosis of intestinal parasites. Am J Clin Pathol 48: 342-346.

15. Bush AO, Lafferty KD, Lotz JM, Shostak AW. 1997. Parasitology meets ecology on its own terms. Margolis et al. revisited. J Parasitol 83: 575-583. doi: $10.2307 / 3284227$

16. Cervone M, Giannelli A, Otranto D, Perrucci S. 2016. Strongyloides stercoralis hyperinfection in an immunosuppressed dog from France. Rev Vét Clin 51: 55-59. doi: 10.1016/ j.anicom.2016.05.001

17. Cicuttin GL, Brambati DF, Rodríguez, JI, Lebrero CG, De Salvo MN, Beltrán FJ, Gury Dohmen, et al. 2014. Molecular characterization of Rickettsia massiliae and Anaplasma platys infecting Rhipicephalus sanguineus ticks and domestic dogs, Buenos Aires (Argentina). Ticks Tick-Borne Dis 5: 484-488. doi: 10.1016/j.ttbdis.2014.03.001 
18. Dantas-Torres F. 2010. Biology and ecology of the brown dog tick, Rhipicephalus sanguineus. Parasite Vector 3:26. doi: 10.1186/1756-3305-3-26

19. González-Acuña D, Guglielmone AA. 2005. Ticks (Acari: Ixodoidea: Argasidae, Ixodidae) of Chile. Exp Appl Acarol 35: 147-163. doi: 10.1007/s10493004-1988-2

20. Gonzalez-Acuña D, Venzal J, SkewesRamm O, Rubilar-Contreras L, Daugschies A, Guglielmone AA. 2004. First record of immature stages of Amblyomma tigrinum (Acari: Ixodidae) on wild birds in Chile. Exp Appl Acarol 33: 153-156.

21. Gorman T, Soto A, Alcaino H. 2006. Parasitismo gastrointestinal en perros de comunas de Santiago de diferente nivel socioeconómico. Parasitol Latinoam 61: 126-132. doi: 10.4067/S0717-77122006000200005

22. Guglielmone AA, Mangold AJ, Luciani CE, Viñabal AE. 2000. Amblyomma tigrinum (Acari: Ixodidae) in relation to phytogeography of CentralNorthern Argentina with notes on hosts and seasonal distribution. Exp Appl Acarol 24: 983-989. doi: 10.1023/ A: 101077552

23. Irwin PJ. 2002. Companion animal parasitology: a clinical perspective. Int J Parasitol 32: 581-593. doi: 10.1016/ S0020-7519(01)00361-7

24. Jimenez-Coello M, Ortega-Pacheco A, Guzman-Marin E, Guiris-Andrade DM, Martinez-Figueroa L, AcostaViana KY. 2010. Stray $\operatorname{dogs}$ as reservoirs of the zoonotic agents Leptospira interrogans, Trypanosoma cruzi, and Aspergillus spp in an urban area of Chiapas in Southern Mexico. Vector-Borne Zoonot 10: 135-141. doi: 10.1089/vbz.2008.0170

25. Lado P, Castro O, Labruna MB, Venzal JM. 2014. First molecular detection of Rickettsia parkeri in Amblyomma tigrinum and Amblyomma dubitatum ticks from Uruguay. Ticks Tick-Borne Dis 5: 660-662. doi: 10.1016/ j.ttbdis.2014.04.02

26. Ley 21020 sobre tenencia responsable de mascotas y animales de compañía. 2017. Ministerio de Salud, Chile. [Internet]. http://www.tenenciaresponsablemascotas.cl/wp-content/ uploads/2018/02/Ley-21.020-sobre-Tenencia-Responsable-de-Mascotas-1.pdf

27. Linardi PM, Santos JLC. 2012. Ctenocephalides felis felis vs. Ctenocephalides canis (Siphonaptera: Pulicidae): some issues in correctly identify these species. Rev Bras Parasitol V 21:345-354. doi: 10.1590/ S1984-29612012000400002

28. López DJ, Abarca VK, Paredes MP, Inzunza TE. 2006. Parásitos intestinales en caninos y felinos con cuadros digestivos en Santiago, Chile. Consideraciones en Salud Pública. Rev Med Chile 134: 193-200. doi: 10.4067/S003498872006000200009

29. Luzio A, Diaz P, Luzio P, Fernández I. 2017. Formas parasitarias gastroentéricas de importancia zoonótica, en heces de perros, recolectadas en plazas de armas de las capitales provinciales de la Región del Bío Bío, Chile. REDVET 18(9). [Internet]. Disponible en: http:// www.veterinaria.org/revistas/redvet/ n090917/091735.pdf

30. Nava S, Mangold AJ, Guglielmone $A \boldsymbol{A}$. 2006. The natural hosts for larvae and nymphs of Amblyomma neumanni and Amblyomma parvum (Acari: Ixodidae). Exp Appl Acarol 40: 123-131. doi: 10.1007/s10493-006-9026-1

31. Nava S, Venzal JM, González-Acuña D, Martins TF, Guglielmone AA. 2017. Ticks of the southern cone of America: diagnosis, distribution, and hosts with taxonomy, ecology and sanitary importance. Academic Press. 372 p.

32. Otranto D. 2015. Diagnostic challenges and the unwritten stories of dog and cat parasites. Vet Parasitol 212: 54-61. doi: 10.1016/j.vetpar.2015.06.002

33. Rishniw M, Barr SC, Simpson KW, Frongillo MF, Franz M, Dominguez JL. 2006. Discrimination between six 
species of canine microfilariae by a single polymerase chain reaction. Vet Parasitol 135: 303-314. doi: 10.1016/ j.vetpar.2005.10.013

34. Rocha FL, Roque ALR, Arrais RC, Santos JP, Lima VDS, Xavier SCDC, Cordeir-Estrela P, et al 2013. Trypanosoma cruzi $\mathrm{TcI}$ and TcII transmission among wild carnivores, small mammals and dogs in a conserva-tion unit and surrounding areas, Brazil. Parasitology 140: 160-170. doi: 10.1017/S0031182012001539

35. Romer Y, Nava S, Govedic F, Cicuttin G, Denison AM, Singleton J, Kelly AJ, et al. 2014. Rickettsia parkeri rickettsiosis in different ecological regions of Argentina and its association with Amblyomma tigrinum as a potential vector. Am J Trop Med Hyg 91: 11561160. doi: 10.4269/ajtmh.14-0334
36. Soulsby EJL. 1987. Parasitología y enfermedades parasitarias en los animales domésticos. $7^{\circ}$ ed. México DF: Interamericana. $823 \mathrm{p}$.

37. Stepek G, Buttle DJ, Duce IR, Behnke JM. 2006. Human gastrointestinal nematode infections: are new control methods required? Int J Exp Pathol 87: 325341.doi: 10.1111/j.1365-2613.2006.-00495.x

38. Tagle I. 1966. Parásitos de los animales domésticos en Chile. Bol Chil Parasitol 21: 118-121.

39. Viera AJ, Garrett JM. 2005. Understanding interobserver agreement: the kappa statistic. Fam Med 37: 360-363.

40. Ybañez AP, Perez ZO, Gabotero SR, Yandug RT, Kotaro M, Inokuma $H$. 2012. First molecular detection of Ehrlichia canis and Anaplasma platys in ticks from dogs in Cebu, Philippines. Ticks Tick-Borne Dis 3: 288-293. doi: 10.1016/j.ttbdis.2012.10.032 